Cahiers d'études italiennes

\title{
Distances et détours : la séduction à l'œuvre chez Alessandro Baricco
}

\section{Yannick Gouchan}

\section{OpenEdition}

\section{Journals}

Édition électronique

URL : http://journals.openedition.org/cei/810

DOI : $10.4000 /$ cei.810

ISSN : 2260-779X

Éditeur

UGA Éditions/Université Grenoble Alpes

\section{Édition imprimée}

Date de publication : 15 septembre 2006

Pagination : 33-44

ISBN : 978-2-84310-086-4

ISSN : 1770-9571

\section{Référence électronique}

Yannick Gouchan, « Distances et détours : la séduction à l'œuvre chez Alessandro Baricco », Cahiers d'études italiennes [En ligne], 5 | 2006, mis en ligne le 15 mars 2008, consulté le 26 mars 2021. URL : http://journals.openedition.org/cei/810 ; DOI : https://doi.org/10.4000/cei.810 


\title{
DISTANCES ET DÉTOURS
}

\section{LA SÉDUCTION À L'CEUVRE CHEZ ALESSANDRO BARICCO}

\author{
Yannick Gouchan \\ Université de Provence - Aix-Marseille 1
}

Le détournement de la vérité qui a longtemps caractérisé le mécanisme de séduction s'est progressivement transformé, au XVIII ${ }^{e}$ siècle, en objectif de conquête amoureuse, pour finir par devenir aujourd'hui un acte de persuasion, de fascination, d'attraction par le langage ou par le regard. Un premier exemple de séduction par le regard chez Baricco se trouve dans le roman Seta:

Hervé Joncour non smise di parlare ma abbassò istintivamente lo sguardo su di lei e quel che vide, senza smettere di parlare, fu che quegli occhi non avevano un taglio orientale, e che erano puntati, con un'intensità sconcertante, su di lui: come se fin dall'inizio non avessero fatto altro, da sotto le palpebre ${ }^{1}$.

L'évolution sémantique du verbe "séduire», qui signifie d'abord "soustraire, tirer à l'écart", selon l'étymologie depuis le verbe latin "subducere", constitue, selon nous, une possible clé de lecture d'une partie de la production narrative d'Alessandro Baricco. C'est dans un ensemble de romans publiés entre 1991 et 2002 qu'il est possible d'étudier certains aspects des rapports amoureux entre les personnages, au cœur desquels les motifs de la distance et du détour permettront d'analyser le mécanisme complexe de la séduction, et notamment des stratégies et des mises en scènes qui en sont le prélude. Ce mécanisme sera même essentiel dans le fonctionnement narratif de certaines œuvres, où la séduction sous-tend l'ensemble du dénouement. Mais les personnages ne sont pas les seuls à être impliqués dans la stratégie de séduction; c'est ainsi que l'écriture si particulière de Baricco, formé, ne l'oublions pas, dans le domaine de la

1. Seta, Milan, Rizzoli, 1996. Nous utilisons l'édition BUR de 1999, p. 25. 
musicologie, utilise les mêmes motifs de la distance et du détour à plusieurs niveaux, aussi bien dans le microtexte que dans une certaine posture de l'auteur face au monde médiatique. Cette brève analyse voudrait donc présenter le fonctionnement de la séduction chez un auteur contemporain, afin de fournir quelques pistes de recherche plus générales sur le vaste problème de la fascination en littérature.

\section{Formes et fonctions de la séduction dans quelques rapports amoureux chez Baricco}

Nous avons annoncé que, dans certains romans de Baricco, il y avait une corrélation entre la logique de séduction et le motif de la distance. En effet, l'intention séductrice commence souvent par l'abolition d'une distance spatiale (et même temporelle, comme on le verra plus loin) entre les êtres, de manière à créer une illusion de proximité, de complicité amoureuse. Nous avançons le terme d'illusion car les deux premiers exemples auxquels nous pensons présentent justement cette virtualité de proximité constituée par un artifice qui tient véritablement de la séduction amoureuse $^{2}$. Ainsi, dans Castelli di rabbia ${ }^{3}$, l'ingénieur Rail en voyage envoie régulièrement des paquets à son épouse, Jun, sans aucune lettre; les paquets contiennent des bijoux qui semblent devenir le message tacite de leur amour à distance, comme pour établir une relation silencieuse et mystérieuse, alors que Rail est parfois infidèle à son épouse, et que cette dernière connaîtra même un bref moment d'inceste avec le fils de son mari. Toutefois, la force de la séduction réside dans cette illusion d'une distance abolie. D'ailleurs Rail a d'abord conquis son épouse en lui donnant l'impression immédiate d'être proche d'elle et de la désirer, juste au moment où elle s'apprêtait à embarquer pour un voyage sans retour, dans un échange de répliques très brèves, où l'humour agit autant que le désir:

E fu lì che vide Jun.

E pensò : io vivrò con lei. [...]

— Io mi chiamo Dann Rail.

- E allora?

— No, niente, volevo dire che... stai per partire?

- Sì.

2. J. Baudrillard parle d'un "piège des apparences", car "la séduction est ce qui ôte au discours son sens et le détourne de sa vérité». De la séduction, Galilée, Paris, 1979, p. 77-78.

3. Castelli di rabbia, Milan, Rizzoli, 1991. Nous utilisons l'édition BUR de 1997. 
- Dove vai?

- E tu?

- Io da nessuna parte. Io non parto.

- E cosa fai qui?

- Sono venuto a prendere qualcuno.

- Chi?

- Te. (Castelli di rabbia, p. 209-210)

Dans le roman Seta, le protagoniste Hervé Joncour reçoit une lettre écrite en idéogrammes japonais qui le place dans l'illusion d'un rapport amoureux avec une jeune fille rencontrée en Asie, alors que la lettre n'est en réalité qu'un artifice de sa propre épouse, Hélène. Cette dernière tente de se rapprocher virtuellement de son époux en organisant à distance une scène érotique, par l'intermédiaire d'une jeune fille japonaise; l'épouse séduit donc son mari au moyen d'une sorte de "donna schermo", lointaine et mystérieuse. La séduction qui fonctionne dans ce roman utilise par contre la distance à la fois géographique et culturelle pour ravir un homme par personne interposée.

Ces exemples montrent un mécanisme séducteur insolite, qui cherche à détourner d'une certaine vérité, mais sans intention de nuire, car c'est une séduction qui doit entretenir un amour conjugal. L'abolition illusoire de la distance par la création d'un amour virtuel se retrouve également dans Oceano mare ${ }^{4}$, avec les lettres enflammées qu'écrit le professeur Bartelboom à la femme qu'il n'a pas encore rencontrée; cependant la séduction virtuelle aboutit dans ce cas à l'abolition d'une distance temporelle, car Bartelboom vit une relation amoureuse en séduisant une femme qui n'existe pas, ou plutôt, qui n'existe que sur le papier des lettres qu'il écrit: l'illusion d'une proximité avec ce personnage épistolaire le pousse même, dans un premier temps, à refuser de se laisser séduire par une véritable femme, Ann Deverià, rencontrée dans une pension. L'un des meilleurs exemples de la séduction visant à abolir la distance entre deux êtres est offert par le personnage de Nina, dans Senza sangue ${ }^{5}$ : une femme âgée cherche visiblement à se rapprocher d'un homme du même âge en engageant une conversation banale sur les billets de loterie ${ }^{6}$, puis elle organise un prélude à la séduction par des regards complices, par une valorisation de la spécificité de l'homme dont elle veut se rapprocher, et enfin par une invitation directe à passer un moment ensemble, par exemple dans un

4. Oceano mare, Milan, Rizzoli, 1993. Nous utilisons l'édition BUR de 1997.

5. Senza sangue, Milan, Rizzoli, 2002.

6. «- Lei è diverso $[\ldots]$ - mi chiedevo se lei avrebbe voglia di venire $[\ldots]$ Ma poi si chinò un po' verso l'uomo e disse: — Venga con me... La prego. ». Ibid., p. 55-56. 
bar. Bartelboom avec ses lettres autant que Nina avec son improbable stratégie utilisent la séduction de manière virtuelle, car la personne à séduire n'existe pas, dans le cas de Bartelboom, ou bien se réduit à une simple vengeance presque dérisoire, dans le cas de Nina (qui semble vouloir exorciser des années d'angoisse lorsqu' elle retrouve l'homme qui a participé au massacre de sa famille bien des années auparavant, mais c'est un homme âgé et totalement vulnérable, désormais inoffensif).

Toujours dans le motif de l'abolition de la distance par la séduction, le cas de la jeune Elisewin, dans Oceano mare, signifie la guérison véritable d'un mutisme et l'accomplissement d'un destin. C'est Adams qui séduit la jeune fille intriguée par le mystère qu'il dégage, et l'abolition de la distance entre les deux êtres se concrétise par un amour physique qui guérit Elisewin de sa maladie, tout en lui ouvrant les portes d'une nouvelle vie, comme un éveil soudain. Tous ces exemples de séduction amoureuse qui ont pour objectif de rapprocher des êtres, de faire croire à une illusion, d'abolir la distance, aboutissent toutefois à des résultats insolites ou inattendus, et c'est bien dans cet aboutissement de la logique de séduction que Baricco arrive parfois à nous surprendre, ou du moins à sortir des traditionnelles histoires sentimentales.

Comme nous l'avons dit, les cadeaux de Rail à Jun n'empêchent pas cette dernière de céder à l'inceste avec le fils adultérin que lui a apporté son mari, Mormy, puis de quitter son époux, comme cela était par ailleurs convenu au moment même où Rail avait séduit Jun la première fois, sur le port. La séduction épistolaire et virtuelle d'une femme par Bartelboom, dans Oceano mare, se conclut de manière tragi-comique par la rencontre avec une vraie femme, Anna Ancher, qui lui semble correspondre à celle qu'il attendait depuis si longtemps. Cependant, au moment où Bartelboom s'apprête à montrer les fameuses lettres à la femme qu'il va séduire, il se rend compte qu'il les avait déjà données à une autre, Maria Luigia, et il se précipite chez elle pour les reprendre, dans une suite de quiproquos et de maladresses qui désamorcent définitivement la stratégie de séduction en la transformant en épisode comique pour le lecteur, par ailleurs détourné du récit amoureux initial: «Ma Bartelboom era uomo probo e giusto, uno di quelli che hanno un certo stile quando si tratta di digerire le bizze del destino. Bartelboom, lui, iniziò a ridere ${ }^{7}$ ».

En ce qui concerne les deux autres grandes stratégies de séduction, celle de l'épouse d'Hervé Joncour (Seta) et celle de Nina face à son ancien bour-

7. Oceano mare, p. 193. 
reau (Senza sangue), le rire cède la place au regret et à la sérénité. En effet, le stratagème de séduction mis en œuvre par Hélène pour attirer son mari était, dès le départ, voué à l'échec, du moins sans aucune possibilité de retour du désir. Joncour ne comprend pas, ou comprendra trop tard, que sa femme l'a séduit par l'intermédiaire d'une jeune Japonaise qu'il croit aimer, comme il ignore qu'elle est aussi à l'origine des lettres en japonais qu'il reçoit: «È uno strano dolore [...] Morire di nostalgia per qualcosa che non vivrai mai ${ }^{8}$ ». La logique de séduction de l'épouse consistait donc à abolir la distance qui la séparait de son mari lorsque celui-ci partait acheter des cocons de vers à soie au Japon. En revanche Nina, désormais âgée, cherche à approcher par une technique de séduction classique (une conversation de plus en plus précise, la recherche de l'intimité dans un bar) l'homme dont elle semble vouloir se venger, Pedro, ancien ennemi de son père et auteur du massacre de sa famille (même si au moment du massacre, qui est décrit dans la première moitié de Senza sangue, Pedro épargne la fillette dissimulée sous le plancher). Ici, la séduction classique relève plutôt d'une logique de vengeance longuement préméditée, par la fragilisation de la cible à atteindre: "Lei è venuta fin qui per cercare me [...] Adesso mi ha trovato ${ }^{`}$ ». Mais la douleur accumulée de Nina se transforme finalement en une véritable séduction de nature amoureuse, destinée avant tout à pacifier l'angoisse, à tourner une page en dépassant la rancœur par la pacification des sentiments; c'est ainsi que Nina, même si elle continue à ne pas comprendre les motivations qui ont amené Pedro à tuer sa famille, pousse la logique séductrice à son terme par une proposition d'amour physique.

Le motif de la séduction dans les romans de Baricco permet, par des moyens détournés, comme l'humour ou la distance géographique, de traiter de manière insolite des problématiques relativement courantes (l'adultère, le dépit amoureux, l'amour conjugal). Mais Baricco nous entraîne aussi vers des problèmes plus complexes et délicats à traiter, comme l'attirance physique pour un membre de sa famille (Jun), l'amour virtuel comme remède à la solitude (Bartelboom), la possibilité d'un désir physique chez des personnes âgées (Nina et Pedro). Ces thèmes très contemporains sont cependant abordés loin de toute analyse psychologique ou d'ancrage dans le monde actuel, car nous verrons plus loin que Baricco cherche justement à séduire son lecteur par la fuite vers des lieux indéfinis et des époques antérieures.

8. Seta, p. 82-83.

9. Senza sangue, p. 58. 
Si la séduction anime de nombreux personnages de Baricco, avec les intentions et les logiques les plus insolites, la structure même des récits en est affectée, au point que deux romans trouvent leur dénouement grâce à l'aboutissement d'un mécanisme séducteur. Ainsi, le récit de Seta se construit autour des voyages d'Hervé Joncour au Japon et de l'évolution de son désir pour la mystérieuse jeune fille. Le dénouement arrive avec la révélation de la stratégie séductrice de l'épouse, mais bien après sa disparition, malheureusement. Madame Blanche, une prostituée, fait comprendre à Joncour que la lettre érotique en japonais qui l'a hanté si longtemps avait été écrite par sa propre femme: «Sapete, monsieur, io credo che lei avrebbe desiderato, più di ogni altra cosa, essere quella donna. Voi non lo potete capire. Ma io l'ho sentita leggere quella lettera. Io so che è $\operatorname{così~}^{10}$ ".

Dans ce cas précis, la séduction a donc bien signifié un détournement intentionnel de la vérité, car Hélène a organisé les conditions pour l'adultère de son mari, dans un élan d'amour conjugal très particulier, parce que l'époux n’a rien soupçonné. Comme pour le dénouement de Seta, la séduction structure celui de Senza sangue. Le premier volet de ce diptyque narratif accumule les détails macabres du massacre de la famille de Nina, tandis que le début du second volet fonctionne au rythme de la logique séductrice de la protagoniste ${ }^{11}$. Toutefois, Nina séduit Pedro pour dépasser sa propre douleur, certes, mais aussi pour tenter de détourner Pedro de sa vérité politique, comme pour séduire un ennemi en le persuadant de l'absurdité de ses actes. Le roman se termine par la pacification obtenue justement par la logique de séduction; comme Elisewin a été guérie de son mutisme, Nina affirme, après avoir fait l'amour avec Pedro : «Io non avrò mai più paura» (p. 98). Tout le roman, et par la même occasion la vie de Nina, semble aboutir vers ce lit où deux vieillards atteignent une forme de sérénité: «Fece quello per cui aveva vissuto» (p. 104).

Ces deux romans trouvent leur dénouement, et leur signification générale, dans le motif de la séduction qui détourne respectivement le thème classique de l'adultère et l'accomplissement d'une vengeance attendue. De plus, le roman Castelli di rabbia avance l'idée d'une séduction perpétuelle entre époux, en l'occurrence Rail et Jun, permettant de relancer le récit à chaque retour de voyage de l'ingénieur; la séduction amoureuse toujours

10. Seta, p. 99.

11. «Lei sembrava si fosse vestita per piacergli». Senza sangue, p. 76. 
recommencée devient alors un élément de dynamique narrative composée de va-et-vient entre les départs et les retours de l'époux (rappelant par ailleurs la même dynamique d'aller et de retour dans Seta, avec les voyages de Joncour qui sont à l'origine de la stratégie séductrice d'Hélène) :

Dopo - dopo era come ricominciare a scrivere da una pagina bianca. Qualsiasi viaggio avesse portato in giro per il mondo il signor Rail, scompariva nel bicchier d'acqua di quella mezz'ora d'amore. Si ricominciava da dove ci si era lasciati ${ }^{12}$.

La mécanique de séduction opérée par les personnages dans les romans de Baricco connaît donc des aboutissements parfois insolites, du moins inattendus. Mais la problématique de séduction littéraire, entendue comme moyen de persuader et de plaire, se révèle encore plus riche de signification dans le rapport entre l'auteur et son lecteur, d'autant plus que les motifs de la distance et du détour se retrouvent également à ce niveau-là de l'analyse.

\section{Éléments pour une étude de la séduction du lecteur chez Baricco}

Le ravissement du lecteur résulte d'une certaine élaboration narrative et linguistique (mais aussi médiatique, comme on le verra plus loin), destinée à séduire; chez Baricco, cette élaboration se fonde notamment sur le détournement, la dissimulation, le mélange, et l'atténuation. Ainsi, un premier élément frappant est la difficulté d'identification du lecteur (italien en l'occurrence) avec les personnages des romans, à cause d'un détournement de la réalité par la création d'univers indéterminés, d'une part et, d'autre part, par le refus quasi permanent (voire systématique) de tout référent à «l'italianité», que ce soit par le choix des noms ou par les références littéraires. L'indétermination semble donc régir l'univers spatial et temporel de Baricco, par une sorte de distance "exotique» avec le monde contemporain. Les lieux des romans sont inventés (Quinnipack, dans Castelli di rabbia) ou sont de véritables "non-lieux», comme la pension Almayer, qui surgit de nulle part dans Oceano mare, ou le paquebot Virginian, sur lequel se déroule une vie entière, dans Novecento ${ }^{13}$. Lorsque les lieux sont un peu plus précis, on remarque cependant une même distance significative par rapport au monde italique: Seta se déroule entre le sud de la France et au Japon, City se déroule dans une métropole améri-

12. Castelli di rabbia, p. 55.

13. Novecento, Milan, Feltrinelli, 1997. 
caine, Senza sangue se déroule dans un pays hispanique non déterminé.

Il en va de même pour le choix des époques, toujours à distance du monde contemporain, à l'exception de City, qui par contre est une véritable fuite dans l'imagination des personnages. Les romans se situent soit dans un XIX siècle imprécis mais tourné vers le progrès et les inventions (Castelli di rabbia et Seta), soit au XX siècle, mais toujours sans précision (l'ère des paquebots transatlantiques dans Novecento, la guerre civile dans un pays hispanophone pour Senza sangue). Le choix des patronymes de personnages correspond aussi à une évasion de la réalité italienne: on trouve ainsi de nombreux noms anglo-saxons (Elisewin, Rail, Gould, Bartelboom, etc.), des noms français (Plasson, Joncour, Hélène, Horeau, etc.) et des noms espagnols (Pedro, Nina), avec l'exception d'un Bonetti, dans Castelli di rabbia.

Un tel détournement systématique de la réalité spatiale et temporelle italienne ne se réduit certes pas à une fuite vers l'imagination pure et le refus d'affronter le présent; d'ailleurs, si Baricco choisit de ne pas ancrer ses récits dans la réalité contemporaine pour mieux faire ressortir des problématiques actuelles (comme la permanence de l'amour conjugal, l'amour entre personnes âgées, l'amour virtuel, entre autres), on peut considérer que ce choix est une sorte de stratégie de séduction, dans la mesure où l'auteur fait contempler le présent par des voies détournées. Cette stratégie littéraire séductrice donne naissance à des personnages hors normes, en proie à l'utopie et parfois aux limites de la folie, comme l'ingénieur Rail, protagoniste de Castelli di rabbia, dont l'auteur précise le caractère insolite, en restant toujours très imprécis sur le plan psychologique: «Non essendo però il signor Rail un uomo come gli altri né Jun una donna come le altre $[\ldots]^{14}$ ». Les personnages fuient leur réalité dans des voyages lointains (Rail, Joncour), et les lieux prennent aussi leur distance avec la réalité, à tel point que leur caractère insolite devient presque familier. C'est par exemple le cas dans Oceano mare, où les personnages mystérieux de la pension Almayer forment un univers clos qui renvoie une certaine image des solitudes et des détresses de notre présent:

C’è qualcosa di... di malato in questo posto. Non te ne accorgi? I quadri bianchi di quel pittore, le misurazioni infinite del professore Bartelboom... e poi quella signora che è bellissima eppure è infelice e sola, non so... per non parlare di quell'uomo che aspetta... quel che fa è aspettare, Dio sa cosa, o chi... È tutto... è tutto fermo un passo al di qua delle cose. Non c'è niente di reale, lo capisci questo ${ }^{15}$ ?

La logique de séduction du lecteur est assez traditionnelle: parler du pré-

14. Castelli di rabbia, p. 29.

15. Oceano mare, p. 92. 
sent par l'intermédiaire du passé, se servir du lointain pour mieux montrer ce qui est proche ${ }^{16}$. Toutefois, Baricco parvient aussi à capturer l'attention du lecteur par d'autres moyens de séduction, et en premier lieu par la nature complexe de ses structures narratives, où l'enchevêtrement des récits provoque un tourbillon saisissant. Castelli di rabbia, Oceano mare et City regorgent de multiples plans narratifs différents, comme dans un labyrinthe d'où les personnages doivent sortir (d'ailleurs les personnages sont à l'image de ce labyrinthe narratif: Rail est prisonnier de son utopie et n'en sortira pas indemne; Gould, l'enfant de City, vit dans le monde parallèle de son imagination féconde; Bartelboom se perd dans sa correspondance amoureuse et cherche à mesurer les limites de l'océan). La manière dont l'ingénieur Rail séduit sa future femme, Jun, représente une sorte de correspondant fictif au pouvoir incantatoire des récits mis en œuvre par Baricco: Rail séduit Jun en lui racontant des histoires, c'est-àdire en l'éloignant de la réalité et en la captivant par un ravissement narratif:

Le storie che ti ho raccontato io. Vedrai che ci casca. Nessuna donna può veramente resistere a quelle storie li. [...] Per due ore il signor Rail raccontò a Jun del vetro. Inventò quasi tutto. Ma alcune cose erano vere. E bellissime. Jun ascoltava. [...]

- Ne hai altre, di storie?... di storie come quella del vetro.

- Un mucchio.

- Ne hai una lunga come una notte?

/ così non ci salì, su quella nave ${ }^{17}$.

La jeune femme se laisse séduire par les histoires plus ou moins vraies de l'ingénieur et décide de ne plus prendre le bateau qui devait l'amener loin de son pays. Le lecteur se retrouve parfois dans la même situation que Jun, en proie volontaire aux affabulations de l'auteur qui multiplie les récits parallèles par des détours incessants, composante essentielle de sa logique de séduction. La multiplication des personnages insolites dans Oceano mare est par exemple brouillée par l'interférence avec le triple récit d'un même naufrage (inspiré de celui de la Méduse) qui partage le livre dans sa moitié. L'accumulation affabulatrice est d'autant plus captivante que d'autres romans de Baricco optent au contraire pour un minimalisme narratif complètement inattendu; c'est ainsi que le modèle du récit essentiel et linéaire, Seta, a été écrit juste après les enchevêtrements d'Oceano mare. Seta et Senza sangue captivent donc sur un tout autre registre, qui rappelle

16. «La séduction a pour objet une différence pour atteindre indirectement l'identité, elle cristallise l'apparence dans laquelle le sujet peut se réfugier". Meyer M., Questions de rhétorique (langage, raison, séduction), Livre de Poche, Paris, 1993, p. 132.

17. Castelli di rabbia, p. 211. 
le poème en prose ou la nouvelle, avec une seule histoire (une histoire de séduction dans les deux cas, mais avec des logiques différentes), jusqu'au dénouement qui apporte la signification de la logique séductrice de deux femmes, Hélène et Nina.

La mise à distance spatio-temporelle et la déstabilisation relative du lecteur par des romans très différents les uns des autres s'accompagnent enfin, et surtout, d'une grande originalité stylistique, en mesure de captiver et de « ravir» le lecteur. La séduction par le discours chez Baricco intervient, selon nous, par l'interférence inattendue et presque maniériste de plusieurs solutions stylistiques simultanément. La formation musicologique de l'auteur se retrouve bien sûr dans certains passages dialogués hérités du mélodrame, avec une suite de brèves répliques, et des répétitions qui tiennent du refrain ou de l'art de la fugue, des silences, etc. Le dialogue de type dramatique est souvent utilisé en interférence avec de longues parties narratives, le contrepoint rythmique devient un élément d'inattendu efficace: ainsi, le chapitre 49 de Seta est une suite de quelques phrases nominales, rappelant les notes d'un scénario ou d'une didascalie, alors que le chapitre suivant est une longue narration au passé simple du voyage de Joncour. Le mélange des genres littéraires peut s'identifier à une logique de séduction, dans la mesure où le discours cherche à plaire en convoquant des solutions traditionnellement incompatibles; dans Oceano mare, l'espace blanc typique du poème en vers voisine avec le dialogue de théâtre et la syntaxe nominale de type énumératif. Les derniers chapitres du roman sont d'ailleurs un exercice de style où le dénouement de l'expérience de chaque personnage est traité dans un genre différent: la poésie, l'épistolaire, le catalogue de tableaux, le récit comique, le dialogue, etc.

La séduction du lecteur ne se limite pas au mélange des genres et à la convocation d'effets musicaux marqués, capables de modifier le rythme de la lecture. Ainsi, la manipulation typographique crée aussi des effets de surprise par le recours à l'italique, aux lettres capitales et aux espaces blancs. L'utilisation de tels effets, accompagnée d'un incessant mélange de modes de discours, semble vouloir emporter le lecteur, et même le ravir, donc le séduire par un détournement, vers des formes littéraires moins fréquentées du public que le roman. Cette séduction est d'autant plus subtile que les limites typographiques et stylistiques entre les différents modes de discours sont assez floues, et l'on passe sans solution de continuité d'un récit au passé simple à un dialogue dramatique puis à un fragment de nature poétique. L'espace de la poésie, le rythme du mélodrame et le langage dramatique deviennent des éléments familiers du discours pour tout lecteur assidu de Baricco, aussi bien dans ses romans les plus complexes 
d'un point de vue narratif (Castelli di rabbia, Oceano mare, City) que dans ses récits épurés et linéaires (Seta, Senza sangue).

L'écriture cherche donc ouvertement à surprendre, à susciter l'insolite, à captiver, mais n'oublions pas que Baricco est aussi un curieux manipulateur éditorial. L'attente calculée entre chaque parution de livre (environ tous les deux ou trois ans) se double d'une véritable stratégie médiatique, dans laquelle l'auteur prend une place importante. Par exemple, la sortie de City en 1999 a été savamment orchestrée sur le seul site Internet de Baricco, sans aucune autre forme de promotion. De plus, le choix littéraire de référents non strictement italiens et la diversité de styles et d'histoires d'un livre à l'autre expliquent le véritable succès critique et commercial de Baricco en France: tous ses romans ont été rapidement traduits depuis la découverte de Seta, en 1997, et la plupart sont déjà disponibles dans une édition économique de poche. Les romans de Baricco sont parvenus à séduire un grand nombre de lecteurs en abordant des thèmes qui ne sont pas nécessairement actuels et contemporains, et en utilisant un style qui multiplie les incursions dans d'autres genres littéraires. Baricco ne dresse pas le portrait d'une génération, mais il est devenu une sorte de "superstar du roman spectacle», où chaque parution suscite son lot de polémiques ${ }^{18}$. Il s'agit bien d'une logique de séduction où tout est mis en œuvre pour un ravissement du lecteur: des récits lointains qui nous parlent du présent, des histoires de séduction amoureuse détournées, une écriture qui puise sa force dans la musique et la poésie, une politique médiatique prudente mais efficace.

L'image de la séduction qu'offrent certains personnages des romans de Baricco se construit à partir de l'illusion d'une complicité amoureuse: un époux pense rester présent dans l'esprit de sa femme en lui envoyant des bijoux, alors qu'elle séduit son beau-fils; une autre femme lance indirectement des invitations érotiques à son mari qui restera longtemps fasciné par une autre; une femme âgée fait des avances à un vieillard qui a tué sa famille; un professeur vit une relation épistolaire virtuelle et ne parvient même pas à convaincre la véritable femme de chair et de sang qu'il rencontre, etc. L'illusion consiste en effet à donner l'impression que la dis-

18. L'expression est de A. Codacci Pisanelli, dans son article «La sostenibile pesantezza del nulla", L'Espresso, 3 juin 1999. 
tance n'existe plus, mais la charge d'incommunicabilité et de solitude qui pèse sur ces rapports amoureux les rend très actuels, pour le moins proches de certaines thématiques narratives contemporaines. La séduction du lecteur fonctionne en revanche par une série de détours référentiels et stylistiques (fuite de la réalité italienne, ouverture vers des genres différents, capacité d'affabulation qui captive), qui se révèlent très efficaces, même si le lecteur de Baricco soupçonne parfois l'auteur d'abuser d'un certain maniérisme littéraire et de profiter d'une popularité médiatique parfois envahissante. 